\title{
Neurophotonics
}

\section{Review of optical coherence tomography based angiography in neuroscience}

Utku Baran

Ruikang K. Wang

\section{SPIE.}




\title{
Review of optical coherence tomography based angiography in neuroscience
}

\author{
Utku Baran ${ }^{\mathrm{a}, \mathrm{b}}$ and Ruikang K. Wang ${ }^{\mathrm{a}, \star}$ \\ aUniversity of Washington, Department of Bioengineering, 3720 15th Avenue NE, Seattle, Washington 98195, United States \\ bUniversity of Washington, Department of Electrical Engineering, 185 Stevens Way, Seattle, Washington 98195, United States
}

\begin{abstract}
The brain is a complex ecosystem, consisting of multiple layers and tissue compartments. To facilitate the understanding of its function and its response to neurological insults, a fast in vivo imaging tool with a micronlevel resolution, which can provide a field of view at a few millimeters, is desirable. Optical coherence tomography (OCT) is a noninvasive method for imaging three-dimensional biological tissues with high resolution $(\sim 10 \mu \mathrm{m})$ and without a need for contrast agents. Recent development of OCT-based angiography has started to shed some new light on cerebral hemodynamics in neuroscience. We give an overview of the recent developments of OCT-based imaging techniques for neuroscience applications in rodents. We summarize today's technological alternatives for OCT-based angiography for neuroscience and provide a discussion of challenges and opportunities. Moreover, a summary of OCT angiography studies for stroke, traumatic brain injury, and subarachnoid hemorrhage cases on rodents is provided. ๑ 2016 Society of Photo-Optical Instrumentation Engineers (SPIE) [DOI: 10.1117/1.NPh.3.1.010902]
\end{abstract}

Keywords: optical coherence tomography; angiography; stroke; traumatic brain injury.

Paper 15044VR received Sep. 29, 2015; accepted for publication Dec. 14, 2015; published online Jan. 20, 2016.

\section{Introduction}

The brain is a delicate yet complex ecosystem for robust behavior, consisting of multiple tissue layers and compartments for different functions. Although the human brain has an average volume of $\sim 1450 \mathrm{~cm}^{3}$ compared to the rodent brain with an average volume of $\sim 450 \mathrm{~mm}^{3},{ }^{1}$ the building blocks are very similar. Neurons and glial cells, the major makeup of the brain, are on a scale of micrometers. Moreover, there is energetic demand during neuronal computation, and the brain's limited energy supply constrains its information processing speed and hence the blood flow dynamics. Rodent models are indispensable in improving our understanding of how blood flow responds to various structures of the human brain, either in a healthy or a diseased state. In this regard, an imaging tool with micron-level resolution, which can provide a field of view at a level of few millimeters with high imaging speed, is desirable for in vivo rodent brain imaging. In doing so, it further requires noninvasive interrogation of the delicate brain tissue.

A number of noninvasive/minimally invasive in vivo imaging modalities have been applied to investigate brain functions in neuroscience. Among them, functional magnetic resonance imaging (fMRI) ${ }^{2}$ and two-photon microscopy (TPM) ${ }^{3}$ are the major tools of choice. For human brain imaging, fMRI has been extensively used to map neural activities at macroscopic scale during complex cognitive efforts such as language, emotion, decision-making, learning, and memory. However, fMRI cannot resolve the changes at microscopic scale, making it difficult to understand complex neural models. On the other hand, TPM allows for detailed examination of neural activities in rodent models, but the standard TPM systems are usually limited with speed and the field of view as well as the penetration depth, and they require the use of contrast agents.

*Address all correspondence to: Ruikang K. Wang, E-mail: wangrk@uw.edu
Accurate visualization and quantification of microvascular networks and tissue perfusion are critical for evaluating therapies that treat neurovascular diseases, ${ }^{4,5}$ such as stroke, traumatic brain injury (TBI), vascular dementia, inflammation, and cancer. Several technologies have been developed to study microvascular networks and tissue perfusion in various applications, including laser speckle contrast imaging, ${ }^{6}$ Doppler ultrasound, ${ }^{7}$ positron emission tomography, ${ }^{8}$ laser Doppler flowmetry, ${ }^{9}$ and photoacoustic imaging. ${ }^{10}$ These techniques have limitations that include one or more of the following: they are invasive, which may disrupt the natural microcirculation; they require the use of contrast agents; they present poor spatial or temporal resolution; they are impractical for rodent imaging settings; they are limited by their shallow imaging depth; they are time consuming; they can only quantify relative changes in blood flow; they are limited to two-dimensional (2-D) superficial images; and others. Therefore, there is still a need for a tool that can noninvasively image the tissue functional vasculature without the requirement of using contrast agents and provide capillary-level resolution images in three dimensions (3-D).

Optical coherence tomography (OCT) is a noninvasive method for imaging 3-D biological tissues with high resolution $(<10 \mu \mathrm{m})$ and without a need for contrast agents. ${ }^{11}$ OCT can image up to a depth of several millimeters at an imaging speed up to $1.6 \mathrm{MHz}$ (line scan rate). ${ }^{12}$ Although the requirements of in vivo rodent brain imaging are difficult to satisfy using the aforementioned traditional technologies, OCT can easily reach the desired speeds and provide high-resolution volumetric images with a large field of view using the available electronics.

Currently, there are two types of OCT implementations, time-domain OCT (Ref. 13) and Fourier-domain OCT (FDOCT). ${ }^{14}$ Even though the idea of using OCT in biomedical imaging applications was proposed in the $1990 \mathrm{~s},{ }^{15}$ it is the 
introduction of FD-OCT that triggered the recent development of a vast array of OCT applications in several fields, e.g., ophthalmology and dermatology. FD-OCT has distinct advantages in its sensitivity and imaging speed as compared to its timedomain counterpart. ${ }^{16}$ The high speed of the FD-OCT systems has made it possible not only to image tissue structural information, but also to investigate functional parameters, for example, in vivo blood flow information. ${ }^{17}$ The inherent contrast for blood flow imaging comes from the endogenous light scattering from moving blood cells; therefore, no exogenous agent is required for imaging.

Earlier review by Boppart summarized the basic OCT technologies used in neuroscience. ${ }^{18}$ Here, we provide an overview of the recent developments of OCT-based imaging techniques for neuroscience applications in rodents, with a particular focus on angiography methods that are developed to visualize functional blood flow within microcirculatory tissue beds in vivo. Because the techniques overlap between different applications, the selections of the methods are not always exclusive to brain imaging. It is the authors' hope that the comparisons provided here can also be of service to the fields outside of neuroscience. The aim has also been to be as inclusive as possible regarding methods but then focus more on the ones that have produced the most promising results. In this paper, we summarize today's technological alternatives in OCT angiography for neuroscience, including a comparison, and provide a discussion of the remaining challenges and opportunities.

\section{Optical Coherence Tomography-Based Angiography Methods}

OCT is a noninvasive imaging technology capable of providing depth-resolved cross-sectional images with micrometer resolution of highly scattering samples, such as biological tissue. ${ }^{11} \mathrm{~A}$ typical OCT system contains a broadband light source that emits light with low temporal coherence. The light is coupled into a Michelson interferometer that divides the light into two arms. One arm, known as the reference arm, transmits the light toward a reference mirror. The second arm, called the sample arm, directs the light toward the tissue of interest. This arm contains an objective lens that focuses the light onto the sample. The light that is backscattered from the tissue structures is recombined with the reference light, producing an interference pattern that is detected by a light detector. To reconstruct the 2-D/ $3-\mathrm{D}$ cross-sectional objects, the beam is scanned across the sample surface.

FD-OCT theory states that the spectral power amplitude of the backscattered wave is equal to the Fourier transform of the axial distribution of the object scattering potential. The advantage of FD-OCT over time-domain OCT is that the sample depth information is obtained in parallel without a need to move the reference mirror, resulting in enhanced system sensitivity to measure the backscattering light, typically with $>20 \mathrm{~dB}$ as compared to its time-domain counterpart. Due to its enhanced sensitivity, it affords faster imaging speed with the limited light power allowed for imaging living tissue (as set by the safety standard for the use of lasers).

FD-OCT contains two versions, spectral-domain OCT (SDOCT) and swept-source OCT (SS-OCT). ${ }^{19}$ In SD-OCT, the optical frequency components are captured simultaneously with a dispersive element and a linear array detector (e.g., line scan camera); however, in SS-OCT, the optical frequency components are captured by a single detector (e.g., photodiode) in a time-encoded sequence by sweeping the frequency of the laser source. A common drawback of SS-OCT is that it contains high phase noise, which is introduced from the cycle-to-cycle tuning and timing variability. ${ }^{20}$ Figure 1 presents a typical implementation of an SD-OCT system.

The temporal and spatial statistics of the OCT signal pattern contain the information of the motion of the scattering particles. Signals coming from the regions with moving particles have a larger variance compared to static tissue. By contrasting these regions through analyzing the phase and/or intensity of complex OCT signals, blood vessels can be identified. There have been several angiography techniques that have been devised based on FD-OCT. These include phase-based methods, which are sensitive to axial flow velocity; intensity-based methods, which are sensitive to dynamic speckle formed by a moving particle; and complex signal-based methods, which are sensitive to both axial flow and dynamic speckles, including those induced by transverse moving particles. Recently, a detailed comparison study between prominent OCT angiography protocols was made

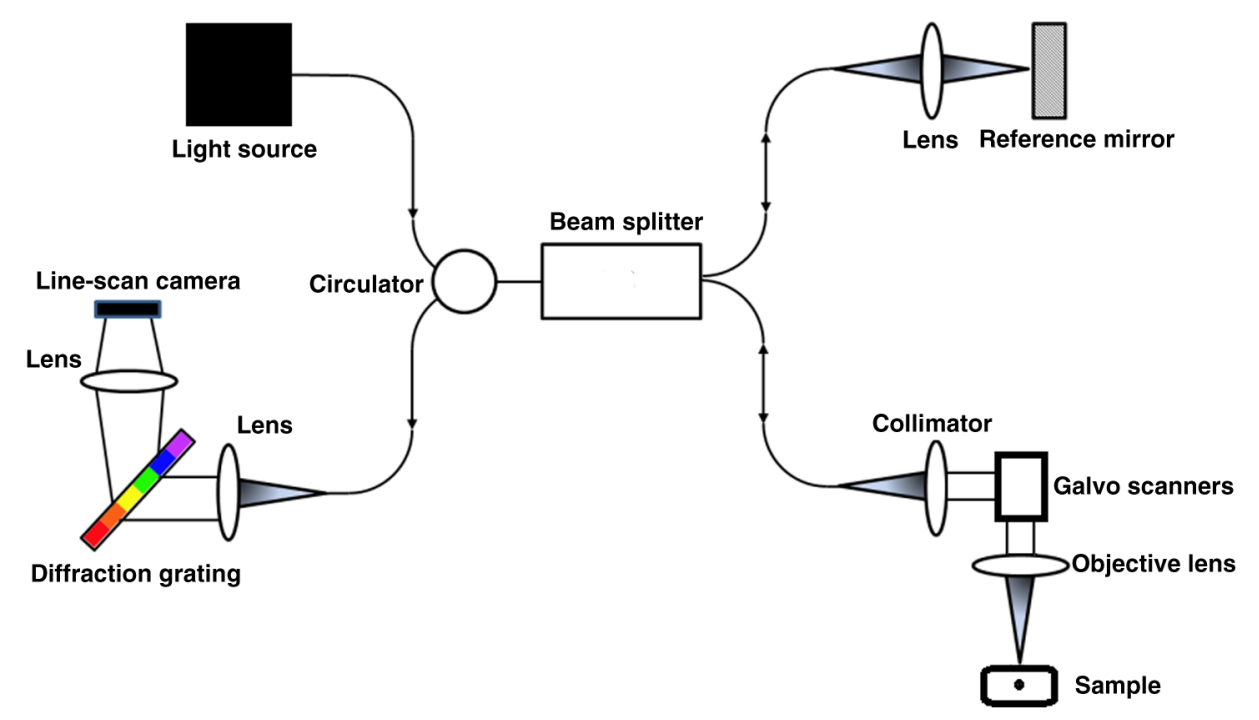

Fig. 1 Typical implementation of an FD-OCT system. 
using a single system, ${ }^{21}$ and brief summaries are provided in the following subsections.

\subsection{Complex Signal-Based Methods}

The complex signal-based methods include optical microangiography (OMAG) ${ }^{22}$ and the complex variance approach. ${ }^{23}$ These methods analyze the A-lines that are acquired at the same location (or at highly overlapping locations) as a time function. A typical way of obtaining the OCT dataset for contrasting blood flow within scanned tissue volume consists of acquiring highly oversampled A-lines when the beam is scanned over the sample.

The sensitivity to blood flow velocities depends on the time interval between the two A-lines that are used for analysis. A shorter time interval is sensitive only for faster blood flows, which are found within relatively large vessels. However, in small vessels such as capillaries, the flow velocity is slow (between 0 and a few $\mathrm{mm} / \mathrm{s}$ ); therefore, a longer time interval between A-lines is required to image them. Hence, these methods might not be the best alternative for time-sensitive cerebral imaging applications if quantifying blood flow with a large dynamic range is required. It is also important to note that if the duration between A-lines is too long, the system will be susceptible to noise due to sample motion and hyper-reflection from static tissue structures.

The analysis of OMAG in the slow axis has been known as ultrahigh sensitive OMAG (UHS-OMAG) ${ }^{24}$ which is sensitive to the slow flow velocities found in capillary vessels. In this method, the signal processing is done between A-lines across different B-frames, which makes it possible not to compromise between the fast imaging speed advantage in FD-OCT and the requirement of a relatively long time interval between A-lines to image smaller vessels, e.g., capillary vessels. To obtain the signals that represent the blood flow, a high-pass filter is used as a differential operation on the complex OCT signals, $C(t, x)$, to eliminate the static tissue signals.

$C_{\text {Flow }}\left(t_{j}, x\right)=\left|C\left(t_{j}, x\right)-C\left(t_{j-1}, x\right)\right|$,

where $j$ represents the index of the B-scan in the slow scan direction, and $x$ is the location of the A-line in the B-scan. The magnitude of differentiated signals in Eq. (1) represents the strength of the flow signals proportional to the particle concentration within the interrogated focal volume. ${ }^{25,26}$ In this implementation, typically a system with a $300 \mathrm{~Hz}$ frame rate is enough to achieve the necessary sensitivity to image slow flows in capillaries because it has been demonstrated that such a system has a sensitivity of $\sim 4 \mu \mathrm{m} / \mathrm{s}$, which is sufficient to image particle Brownian motion. ${ }^{24}$ Another variation of the OMAG scanning pattern is to perform repeated B-scans (typically two or more) at one transverse location (i.e., fast axis), then move to the next transverse location to do the same until the last location is reached in the slow scan axis. ${ }^{27}$ At each location, the analysis of Eq. (1) is applied to the repeated B-scans to extract the motion signal due to moving particles, for example, red blood cells (RBCs). A similar algorithm was also proposed by Srinivasan et al., ${ }^{28}$ in which the angiogram was obtained by taking the difference of the weighted OCT signals acquired between adjacent B-scans.

\subsection{Phase-Based Methods}

Phase-based methods evaluate the axial velocity of flowing particles within a scattering medium, such as blood cells within biological tissues, by utilizing the well-known Doppler effect. This is done by analyzing the phase difference between adjacent A-lines ${ }^{29}$ similar to complex signal-based methods. The phase difference between sequential A-lines, $\Delta \varphi(z)$, is linearly related to the flow axial velocity $\left(v_{\text {axial }}\right)$ by

$v_{\text {axial }}(z)=\frac{\lambda \Delta \varphi(z)}{4 \pi n \Delta t}$,

where $\Delta t$ is the time interval between adjacent A-lines and $n$ is the index of refraction of the tissue. Accordingly, a longer $\Delta t$ is required for detecting slow velocities, which can be challenging for capillary flow detection.

Several variations of phase-based methods for angiography have been reported where the absolute value of the phase difference, ${ }^{30}$ squared phase difference (i.e., Doppler power), ${ }^{31}$ or the variance of several adjacent A-line phase differences ${ }^{32}$ are used. The comparison between different methods can be found here. ${ }^{33}$ The recent work of You et al. ${ }^{34}$ showed great promise in quantitative capillary flow detection by using a phase summation method and vessel tracking. Moreover, Doppler optical microangiography (DOMAG) ${ }^{35}$ has been proposed to improve the performance of phase-based methods. DOMAG utilizes OMAG to separate the static from the dynamic signal, then analyzes the phase difference between adjacent A-scans. In this way, the noise produced by the optical heterogeneous properties of the sample is reduced. Moreover, a variation of DOMAG has recently been introduced ${ }^{36}$ by selectively skipping A-lines, and therefore the duration of $\Delta t$, for analyzing the Doppler signal. This method is simple and straightforward, but important in the investigation of impaired blood flow within microcirculatory tissue beds because it can provide Doppler analyses over a wide velocity range from capillaries to arterioles and venules, providing useful information to aid in the assessment of whether the blood flow within the injured region of interest is compromised or has ceased. Overall, two main factors cause the performance of phase-based methods to deteriorate: (1) the optical heterogeneity of the sample ${ }^{37}$ and (2) the phase instability caused by the light source or the sample motion artifacts. ${ }^{38}$

\subsection{Intensity-Based Methods}

Using only intensity amplitude to generate angiography maps of tissue beds has been very popular since phase noise is a major challenge to overcome in complex- or phase-based methods. On the other hand, in cases where the flow induces the change only in the phase of the OCT signal, removal of the phase information might have a negative effect. The intensity amplitude-based methods include correlation mapping, ${ }^{39}$ split-spectrum amplitude decorrelation, ${ }^{40}$ speckle variance, ${ }^{41}$ and correlation masked speckle variance ${ }^{42}$ techniques.

These techniques take advantage of the strong speckle effect in the vicinity of functional vascular regions compared to nonflow regions (bulk tissue). In fact, this feature of dynamic speckle within OCT signals due to moving particles was first discovered by Barton and Stromski ${ }^{43}$ and utilized to image blood flow within tissue beds. By calculating correlation coefficients or speckle variance across the pixels, blood vessel morphology can be visualized. Contrast is achieved since flow 
regions exhibit low correlation coefficient values and high speckle variance values, while the bulk tissue gives high correlation and low speckle variance values. Dense scanning is usually required for the success of these techniques.

\subsection{Capillary Flux Mapping Methods}

RBC flux and velocity measurements in capillaries are challenging to make using the above common angiography methods, due to the low hematocrit counts in the capillaries and the angle dependency of Doppler measurements. To tackle these limitations, Ren et al. ${ }^{44}$ developed a particle counting Doppler OCT technique that is capable of imaging RBC flux and velocity within a single capillary in the rodent cortex. The authors counted the Doppler phase transients induced by the passage of an RBC through a single capillary to estimate the RBC flux $(\sim 19 / \mathrm{s})$ and the average $\mathrm{RBC}$ velocity $(\sim 0.72 \mathrm{~mm} / \mathrm{s})$.

Moreover, researchers at Martinos Center for Biomedical Imaging developed similar methods to acquire RBC flux and velocity values at multiple capillaries. The first method ${ }^{45}$ monitors the time course of the OCT intensity signal at the capillaries where individual RBC passage is believed to cause a peak in the signal, and the width of the peak would be negatively correlated with the speed. These can be captured by fitting Gaussian functions to these peaks. The accuracy of this method is tested by a traditional stripe pattern-based estimation. The caveat of this method is the long imaging time due to the need for a large number of measurement points.

As an alternative, the same group also proposed a technique called statistical intensity variation (SIV) imaging ${ }^{46}$ where more RBCs passing from a certain location will create a statically higher SIV. Here, SIV between two consecutive frames can be computed by

$$
\operatorname{SIV}(z, x, y)=\frac{E\left[I\left(z, x, t_{2} ; y\right)-I\left(z, x, t_{1} ; y\right)\right]^{2}}{E\left\{\frac{1}{2}\left[I^{2}\left(z, x, t_{2} ; y\right)+I^{2}\left(z, x, t_{1} ; y\right)\right]\right\}},
$$

where $I\left(z, x, t_{1} ; y\right)$ and $I\left(z, x, t_{2} ; y\right)$ are the first and second Bscans at a given Y-position, respectively, and $E[]$ denotes ensemble averaging, which can be implemented by averaging over neighboring voxels and/or repeated volumes. This ensemble averaging minimizes the stochastic speckle effect in the OCT intensity. The computed SIVs are then compared in various capillary segments to estimate RBC flux in these segments. Although this technique enables fast acquisition of capillary flux data, it requires a complicated 3-D Hessian matrix analysis-based capillary vectorization and, thus, is computationally demanding.

Moreover, Wang and Wang ${ }^{25}$ demonstrated an autocorrelation method for mapping transverse particle-flow velocities by estimating the dwell time of flowing particles crossing the probe beam. They showed that the slope of the normalized autocorrelation function of the backscattered light is proportional to the transverse velocity. The same authors also applied another method analogous to the widely used fluorescence correlation spectroscopy technique, ${ }^{47}$ which correlates the fluctuations of the OCT signals to the fluctuations of the particle numbers flowing through the detection volume. ${ }^{48}$ These techniques have been verified with flow phantoms and can be applied for RBC flux and velocity measurements in rodent cortex. Moreover, Srinivasan et al. ${ }^{49}$ used complex field autocorrelation functions as a metric for capillary velocities in rodent cortex and correlated with the velocity measurements obtained from TPM. In contrast to Refs. 25 and 48, the analysis was performed on A-lines along the slow axis of repeated B-scans.

\section{Optical Coherence Tomography Imaging in Healthy Rodent Brain}

The cortex of the brain is layered, and each layer contains various neuron subtypes and different input or output connections to the other regions of the central nervous system. The blood supply to the cortex includes large vessels over the surface of the brain and smaller diving vessels that penetrate almost perpendicular to the surface to feed the dense networks of capillaries in deeper layers. ${ }^{50}$ The capillary beds deliver blood to vital parts of the cortex that are metabolically active during neuronal firing. The signals observed in superficial arteries and veins are related to the in-flow and out-flow of blood to these capillary beds and can have different behavior from their capillary counterparts. The dynamics of the capillary responses are most interesting given that they are expected to be more closely related to the functional behavior of the responding neurons.

The first demonstration of FD-OCT-based angiography on a rodent cerebrovascular model was made in 2007 using the OMAG technique. ${ }^{22}$ Here, Wang et al. demonstrated the capabilities of the OMAG technique for visualizing microvasculature in the brain cortex of mouse models using 820- (Ref. 22) and $1300-\mathrm{nm}^{51}$ central wavelength light sources. Due to the enhanced imaging depth provided by the latter light source, OMAG was able to visualize pial vessels with the scalp left
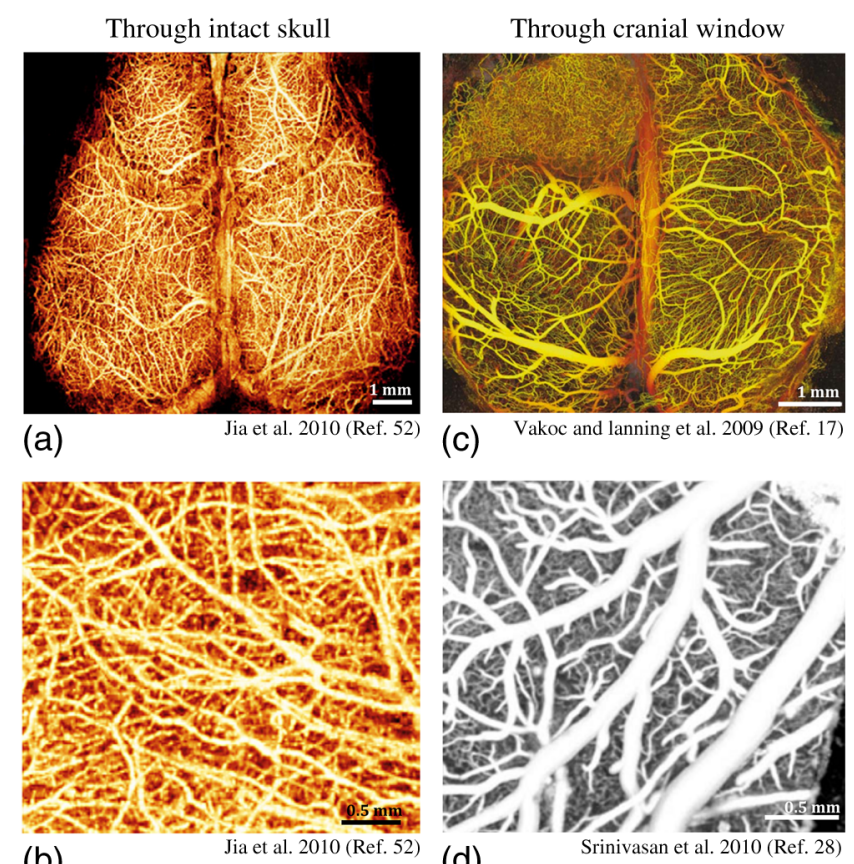

(b)

(c)

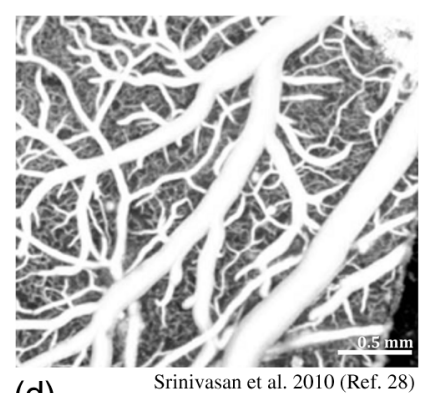

(d)

Fig. 2 (a) and (b) OCT angiography images of mouse cerebral cortex through skull using UHS-OMAG. Images are shown in en face maximum intensity projection (MIP) of volumetric angiography data. ${ }^{52}$ (c) The depth-projected en face MIP of volumetric vasculature within the first $2 \mathrm{~mm}$ of mouse brain bearing a human glioblastoma tumor imaged with phase-based OCT angiography through a cranial window. Depth is denoted by color: yellow (superficial) to red (deep). ${ }^{17}$ (d) En face MIP of vasculature using high-pass filtered intensitybased OCT angiography through a cranial window. ${ }^{28}$ 
intact. ${ }^{51}$ However, due to the sensitivity limitation in the original OMAG protocol, capillaries were not distinguished clearly.

Vakoc et al. ${ }^{17}$ presented a comprehensive view of mouse cerebral vasculature bearing a human glioblastoma tumor through a cranial window using phase-based OCT angiography [Fig. 2(c)] and combined these results with multiphoton microscopy images. Here, the smallest superficial capillaries were visualized with multiphoton microscopy, and OCT angiography provided a superior view of vessels deeper within the central regions of the tumor and in regions where fluorescent tracers leaked outside of vessels. However, a relatively slow frame rate $(\sim 16 \mathrm{~Hz})$ was used to be able to capture slow flow in this work, which can be problematic for time-sensitive experiments.

The following year, real-time imaging of cerebral microvasculature became popular using high-pass filters on complex signals, as explained in Sec. 2.1. Thanks to the higher sensitivity provided by UHS-OMAG, Jia et al. ${ }^{52}$ managed to visualize cerebral microvasculature up to the capillary level within the meninges and cortex in mice with the cranium left intact, as shown in Figs. 2(a) and 2(b). Using a thrombotic mouse model, longitudinal measurements of meningeal vascular responses to the insult and the responses in the cortex are provided. ${ }^{53}$

In addition, Srinivasan et al. ${ }^{28}$ utilized high-pass filtered intensity-based OCT angiography for rapid comprehensive imaging of the surface pial vasculature and capillary bed in the cerebral cortex through a cranial window in $\sim 12 \mathrm{~s}$ [Fig. 2(d)]. Benefiting from the higher-quality images provided by the cranial window, the authors were able to study the changes in vessel diameters and connections during hypercapnia. Similar work also monitored microcirculation responses during hypoxia through the intact skull. ${ }^{54}$

Although high-quality angiograms are useful to detect changes in the microvasculature, quantitative cerebral blood flow (CBF) information is needed in most cases to draw clinical implications. Srinivasan et al. ${ }^{55}$ utilized Doppler OCT to calculate total $\mathrm{CBF}$ and velocity changes in the rat cortex. By the use of a popular method in the Doppler ultrasound community, ${ }^{56}$ the authors demonstrated that the total blood flow within a vessel can be evaluated by integrating en face Doppler signals, which does not require explicit knowledge of Doppler angles. Their measurements in rat somatosensory cortex yielded an average blood flow of $\sim 1.07 \times 10^{-3} \mathrm{ml} / \mathrm{mm}^{2} / \mathrm{min}$, which is consistent with the results acquired using autoradiography in similar rats and anesthesia conditions. ${ }^{57}$ Using this method, they also compared CBF changes with simultaneous hydrogen clearance in rats. ${ }^{58}$

However, typical Doppler OCT methods suffer from limited dynamic velocity range and necessary sensitivity to measure capillary flows (see Sec. 2.2). To overcome the first limitation, Shi et al. ${ }^{36}$ proposed a wide velocity range DOMAG to visualize fast and slow flows within one scan in a mouse cortex. Using this method, the authors demonstrated that using multiple velocity ranges, a comprehensive map of blood flow dynamics can be acquired without sacrificing sensitivity for slow flow or dynamic

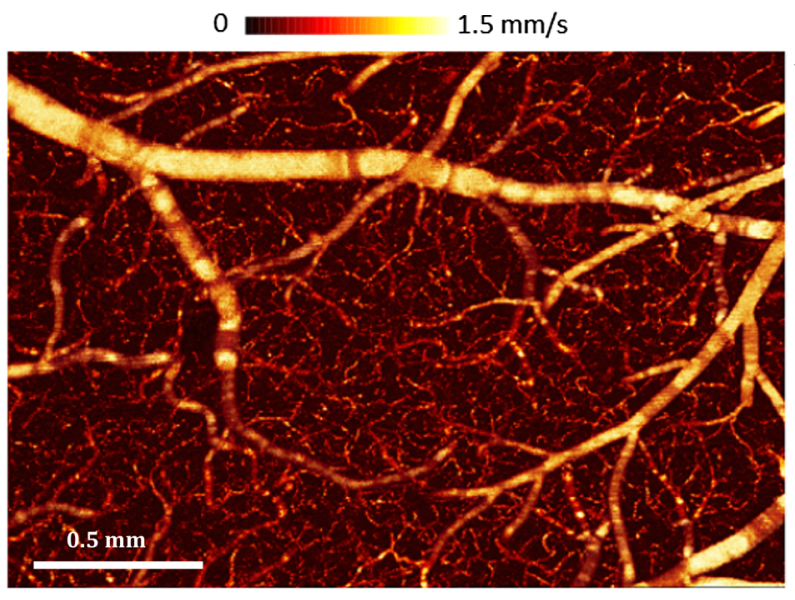

You et al. 2014 (Ref. 34)

(a)

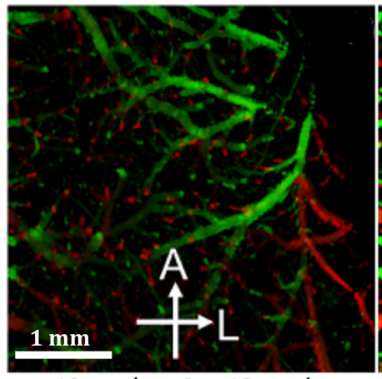

(b)

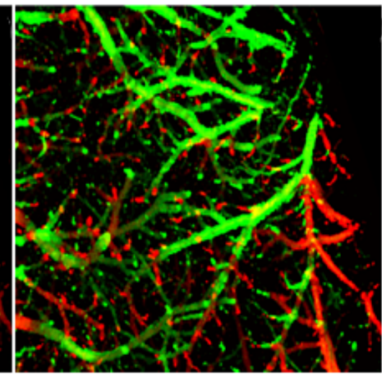

$-6 \mathrm{~mm} / \mathrm{s} \quad 0 \quad 6 \mathrm{~mm} / \mathrm{s}$
Shi et al. 2013 (Ref. 36)

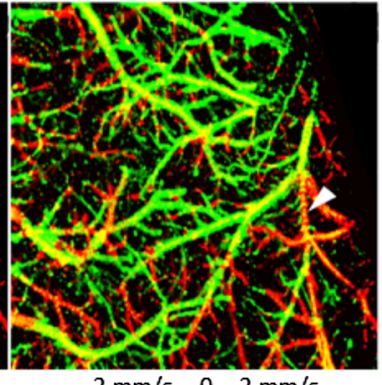

$-2 \mathrm{~mm} / \mathrm{s} \quad 0 \quad 2 \mathrm{~mm} / \mathrm{s}$

Fig. 3 (a) En face MIP image of volumetric Doppler OCT data of the mouse cerebral cortex up to 1-mm depth, utilizing phase summation and angle correction techniques. ${ }^{34}$ (b) En face MIP image of volumetric bidirectional wide velocity range DOMAG of a mouse brain in vivo. The velocity maps are displayed with different velocity ranges. Yellow represents the phase wrapping when the flow is faster than the detection range. ${ }^{36}$ 
range for fast flow [Fig. 3(c)]. Moreover, You et al. ${ }^{34}$ presented a phase summation method to enhance slow capillary flow detection sensitivity using Doppler OCT [Fig. 3(b)]. Using this method, they studied the blood flow response in the cortex to cocaine administration up to the capillary level. Accordingly, they discovered a decrease of blood flow velocity within capillaries from $0.31 \mathrm{~mm} / \mathrm{s}$ in control mouse (consistent with Ref. 59) to $0.15 \mathrm{~mm} / \mathrm{s}$ in the chronic cocaine mouse even though there were no significant changes in capillary density.

RBCs pass one by one in cerebral capillaries, often with a large separation between them. This makes it hard to accurately quantify the RBC flux in capillaries using Doppler OCT methods. To address this problem, Lee et al. proposed new methods recently ${ }^{45,46}$ (see Sec. 2.4). Benefiting from these methods, they mapped heterogeneous flux distribution in cerebral capillaries with the mean RBC flux of $\sim 20 \mathrm{RBCs} / \mathrm{s}$ in healthy rat brain (Fig. 4), similar to Ref. 44. The dynamic range of RBC flux is a function of the time gap between measurement points, in this case. the frame rate. The upper limits in the measured capillary $\mathrm{RBC}$ flux in these studies $(40 \mathrm{RBCs} / \mathrm{s})$ were relatively lower than those in the literature, where the reported values range from 40 to $45 \mathrm{RBC} / \mathrm{s}^{60}$ to $80 \mathrm{RBC} / \mathrm{s}^{59}$ Considering this high variability across the literature, possibly due to the differences in the animal species, anesthesia, and physiology, higher frame rates should be utilized for more accurate RBC flux estimations.

Over the last few years, multimodal imaging systems have been proposed to combine the advantages of OCT with other imaging modalities. Dziennis et al. ${ }^{61}$ used dual-wavelength laser speckle contrast imaging (DWLS) as a guiding tool for OMAG. Here, DWLS [Fig. 5(a)] enabled rapid prediction of the infarct area and hemoglobin oxygenation over the whole mouse brain, and the OMAG system [Fig. 5(b)] provided detailed information about blood perfusion dynamics down to the capillary level in a region of interest pertaining to ischemia. Recently, Gagnon et al. ${ }^{62}$ combined two-photon laser scanning microscopy (TPLSM) angiography with Doppler OCT to acquire a more accurate 3-D volumetric blood flow map by reconstructing the TPLSM angiogram from the model with constraints from the Doppler OCT measurements [Figs. 5(c)-5(e)]. Moreover, TPLSM is also utilized in other studies to confirm the accuracy of capillary measurements of OCT angiography images. $^{17,63}$

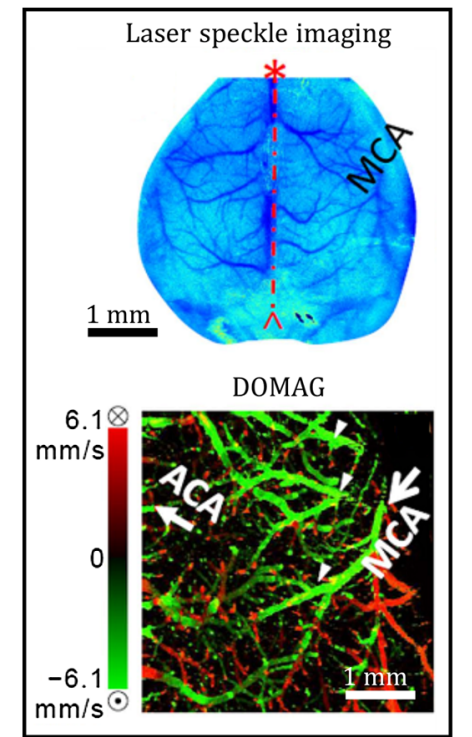

(a)
Dziennise et al. 2015 (Ref. 61)

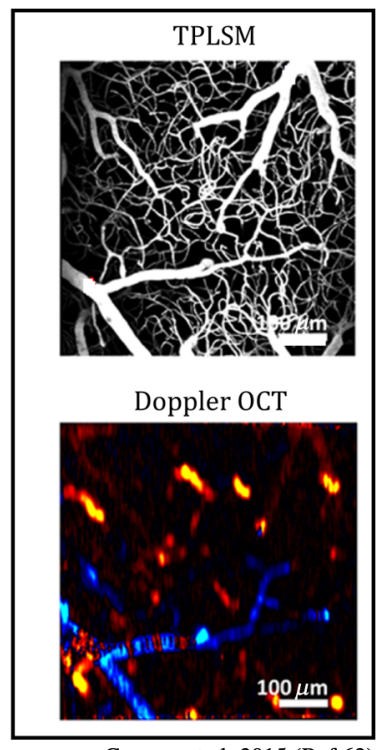

(b)

Gagnon et al. 2015 (Ref.62)
Fig. 5 (a) A combined DWLS and OCT system is used for monitoring of microvasculature in mouse cortex through intact skull. DWLS image shows the microvasculature with a large field of view. En face MIP of DOMAG image provides a closer look. ${ }^{61}$ (b) Combined TPLSM angiography with Doppler OCT for blood flow imaging in the mouse cortex: TPLSM angiogram provides a detailed view of the capillaries. En face MIP of Doppler OCT angiogram provides an RBC velocity map within the larger vessels. ${ }^{62}$

\section{Optical Coherence Tomography Imaging in Rodent Brain During Stroke}

Stroke is caused by blockage (ischemic) or rupture (hemorrhagic) of a blood vessel within the brain. This injury leads to a series of functional and structural changes that may result in an infarct or a peri-infarct brain tissue. The peri-infarct tissue region, also called a penumbra, is potentially recoverable if it can be identified and treated appropriately. Cells often work in synchrony as a neurovascular unit in response to stroke. To improve the understanding of this complex response and to develop better strategies to help recover from stroke, OCT angiography has been utilized over the last few years to study realtime vascular dynamics in vivo on rodents after stroke.
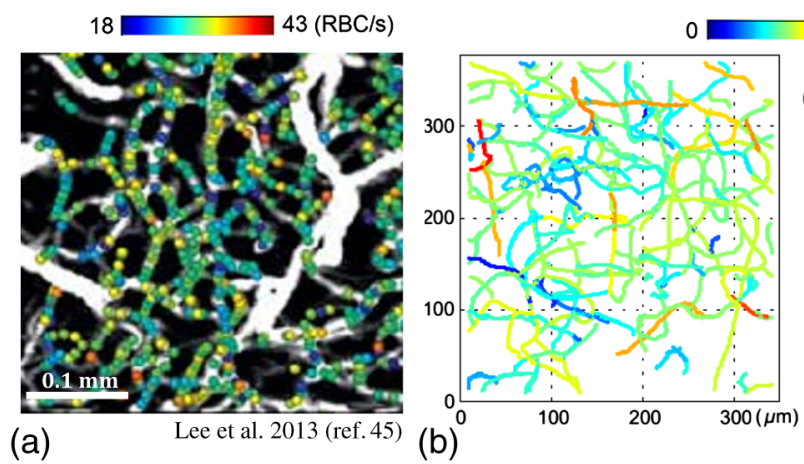

(b)

Fig. 4 (a) RBC flux in rat cortex capillaries is shown as color spots on the en face MIP of the 3-D angiogram. ${ }^{45}$ Flux is estimated by monitoring the time course of the OCT intensity signal. (b) 3-D RBC flux map of the capillary network in rat cortex using SIV method. ${ }^{46}$ The bar graph on the right side presents the RBC flux histogram of the identified 178 capillaries. 
The most common stroke model used on rodents is the intraluminal middle cerebral artery occlusion (MCAO). ${ }^{64}$ Accordingly to this model, a nylon filament with a silicone-coated tip is inserted into the right internal carotid artery via the external carotid artery until it blocks the middle cerebral artery. Three years after the introduction of OMAG, Jia and Wang ${ }^{65}$ presented the first preliminary stroke study using OCT angiography [Figs. 6(a)-6(c)]. They imaged both ipsilateral and contralateral sides of MCAO through intact skull on mice. Here, it was observed that the occlusive components of ischemic stroke still existed in the ipsilateral side after reperfusion from MCA. A more comprehensive study of vasodynamics after focal stroke was presented by Srivinasan et al. ${ }^{66}$ The authors used a multiparametric OCT platform for longitudinal imaging of ischemic stroke in mice, through thinned skulls [Figs. 6(d)-6(f)]. Capillary nonperfusion, CBF deficiency, and altered cellular scattering were quantified and correlated with histology. On the other hand, Yu et al. ${ }^{67}$ applied photodynamic therapy to study dynamic blood circulation before and after a localized ischemic stroke in a mouse model using Doppler OCT.

Developments in OCT angiography paved the way to several discoveries in stroke recovery research over the last couple of years. Berny-Lang et al. managed to increase neurological outcome after stroke by administrating thrombin mutant W215A/ E217 to mice. ${ }^{68}$ Later, Sun et al. utilized UHS-OMAG to reveal that conditional ablation of neuroprogenitor cells does not affect the functional blood flow during reperfusion $1 \mathrm{~h}$ after stroke, although it impeded the recovery of cognitive function. ${ }^{69}$ Moreover, another study showed that impaired collateral status due to diabetes leads to a worse stroke outcome, and modulation of collateral flow has beneficial effects on these subjects. ${ }^{70}$
Recently, Baran et al. investigated the vasodynamics of pial and penetrating arterioles after stroke on mouse using UHSOMAG. ${ }^{71}$ They discovered that the arteriolar-arteriole anastomosis (AAA) plays a significant role in active vasodilation of pial arterioles such that the ones away from anastomosis shrink in diameter, whereas the ones close to anastomosis dilate during stroke. In other words, AAA compensates a significant amount of blood to the ischemic region, whereas poor blood perfusion occurs at the regions away from AAA connections, leading to ischemia (Fig. 7).

\section{Optical Coherence Tomography Imaging in Rodent Brain After Injury}

TBI often causes alterations in CBF, which are thought to influence secondary pathophysiology and neurologic outcome in the human brain. Inadequate $\mathrm{CBF}$ is an important contributor to mortality and morbidity after TBI; ${ }^{72}$ however, it is poorly studied. The ability to noninvasively map the variations of $\mathrm{CBF}$ over a long period of time using OCT angiography would contribute to the understanding of the complex mechanisms related to TBI recovery.

The first demonstrations of OCT angiography for monitoring TBI recovery were conducted by Jia et al. ${ }^{73}$ and Cetas et al. ${ }^{74} \mathrm{Jia}$ et al. ${ }^{73}$ demonstrated that OCT angiography can be used to capture the capillary response to a TBI in the cortex of a mouse model for a period of 10 days with the cranium left intact. In this study, TBI was induced by puncturing a hole of $1.5-\mathrm{mm}$ depth into the cortex through the skull. A typical series of UHS-OMAG CBF images is shown in Fig. 8. Figure 8(a) is the control image, where the capillaries can be clearly observed. At $30 \mathrm{~min}$ after TBI, a reduction is seen in CBF (cerebral ischemia) over the entire cortex in the UHS-OMAG image [Fig. 8(b)].

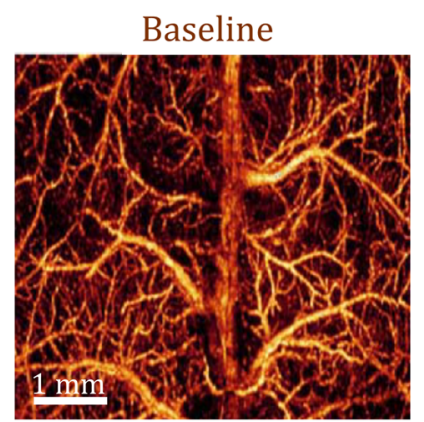

(a)

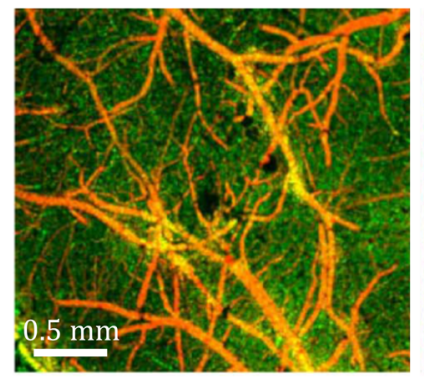

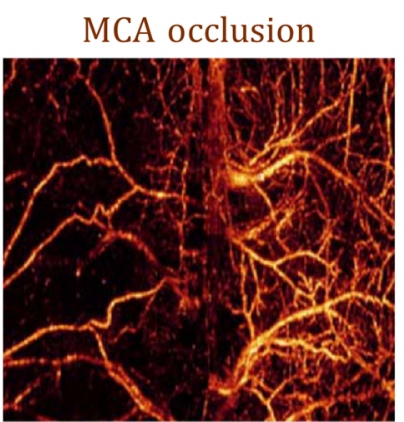

(b)

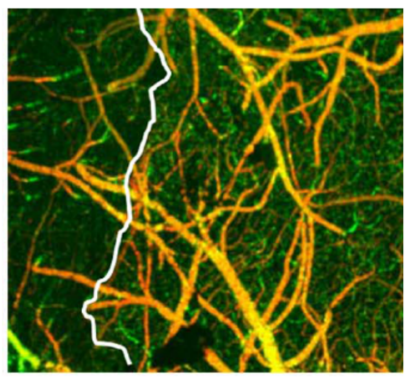

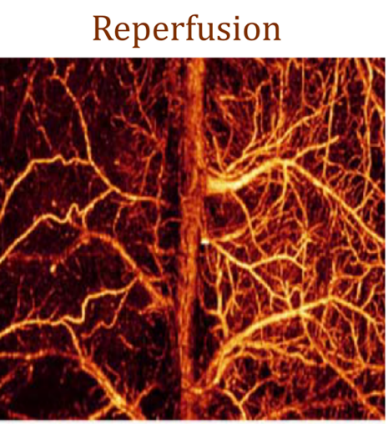

(c)

Jia et al. 2011 (Ref. 65)

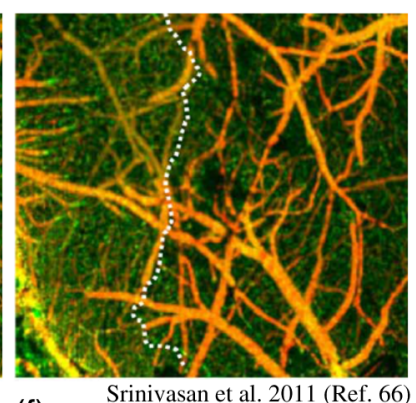

(d)

(e)

(f)

Srinivasan et al. 2011 (Ref. 66)

Fig. 6 Volumetric OCT angiography imaging of the cortex during ischemic stroke. En face MIP of UHSOMAG images (a) during baseline, (b) progressive focal ischemia developed during MCAO, and (c) 30 min after onset of reperfusion. ${ }^{65}$ En face MIP of OCT angiograms (d) at baseline, (e) during $\mathrm{MCAO}$, and (f) $60 \mathrm{~min}$ after reperfusion. During MCAO (e), a capillary nonperfused region is apparent, as marked with a solid white line. ${ }^{66}$ 


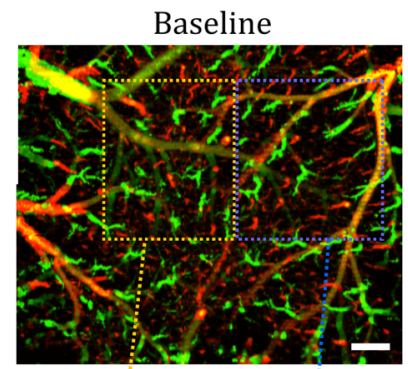

(a)

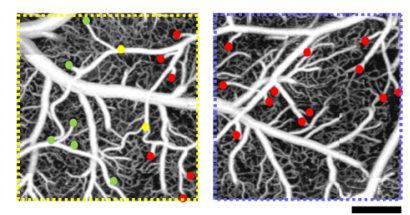

(d)

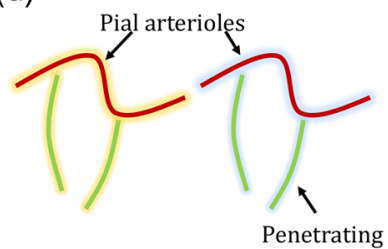

(g)

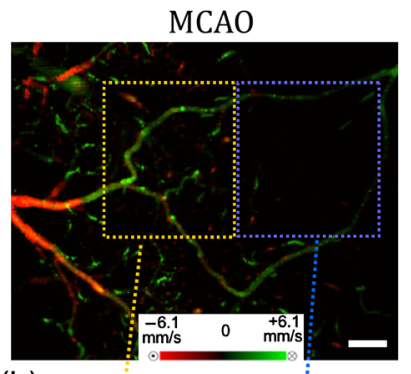

(b)

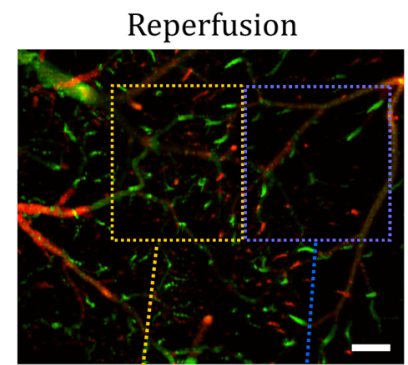

(c)

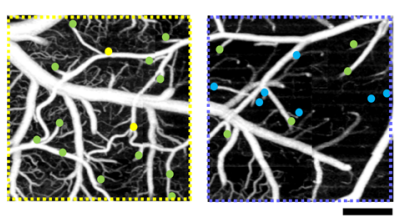

(e)

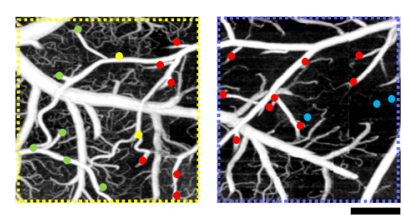

(f)

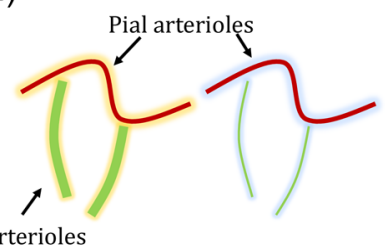

(i)

Fig. 7 Comparison between regions where AAA is relatively stronger or weaker during stroke: ${ }^{71}$ DOMAG results for (a) basal, (b) during MCAO, and (c) after reperfusion conditions, respectively. Strong AAA area is marked with a yellow dashed box and weak AAA area with a blue dashed box. OMAG comparison between strong and weak AAA ROIs for (d) basal, (e) during MCAO, and (f) after reperfusion conditions, respectively. Red, green, and yellow dots in (g) through (i) correspond to MCA, ACA, and AAA T-junction sourced arterioles. Blue dots correspond to the diving arterioles that are at not-detectable level compared to basal condition. Cartoon representations of the lumen diameters of pial and penetrating arterioles for (g) basal, (h) during MCAO, and (i) after reperfusion conditions, respectively. Scale bar $=0.3 \mathrm{~mm}$.

The reduction of $\mathrm{CBF}$ may be due to the subarachnoid hemorrhage $(\mathrm{SAH})$ caused by the external injury that leaves blood in the space between the meninges and cortex near the TBI site (pointed by the green arrow). From days 4 to 7, new blood vessels start to appear in the traumatic region, indicating neovascularization [Figs. 8(d)-8(f)].
Moreover, the same group used OCT angiography to study the therapeutic effect of soluble epoxide hydrolase (sEH) gene deletion in revascularization after TBI. ${ }^{75}$ The results showed that the restoration of blood volume at the lesion site was more pronounced in sEH knockout mice than in wild-type mice. These OCT angiography measurements were also confirmed by histology.

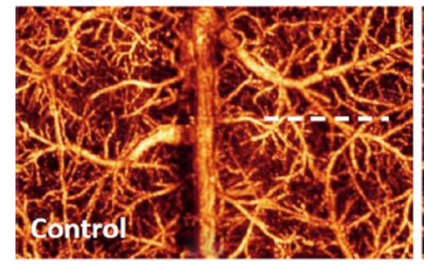

(a)

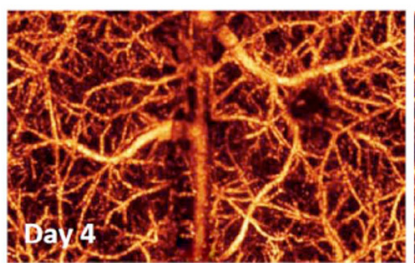

(d)

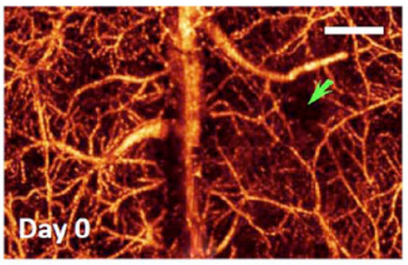

(b)

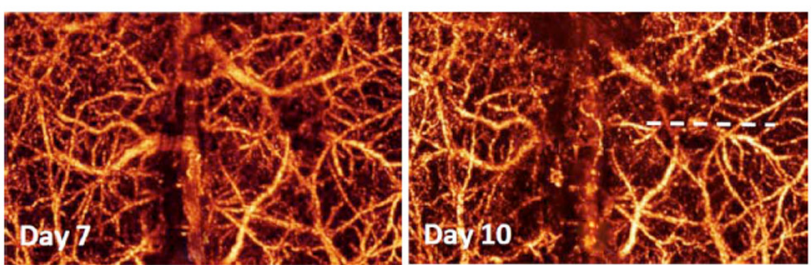

(e)

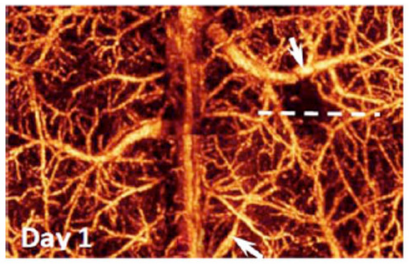

(c)

Fig. 8 En face MIP of volumetric OCT angiography images of the cortex during TBI in mice in vivo at different time points. ${ }^{73}$ Compared to baseline (a), progressive vessel regulation (vessels indicated by white arrows) and neovascularization at traumatic areas develop during TBI rehabilitation. The site of injury is indicated by the green arrow. The image size in (a) through (f) is $\sim 7.2 \times 4.2 \mathrm{~mm}^{2}$. Scale bar $=1.0 \mathrm{~mm}$. 
Subarachnoid space plays critical roles in both the regulation of intracranial pressure (ICP) and maintenance of the brain's extracellular environment. ${ }^{76}$ Blood leakage into that space, mostly triggered by $\mathrm{TBI}$, is called $\mathrm{SAH}$, and it can cause serious complications. ${ }^{77}$ Unfortunately, these complications remain poorly understood. The first study of SAH using OCT angiography focused on the role of the rostral ventromedial medulla (RVM) in modulating cerebral perfusion at rest and following an experimental SAH in rats. ${ }^{74}$ Changes in CBF were measured using laser Doppler flowmetry and OCT angiography. RVM was activated or inactivated using a gamma-aminobutyric acid A receptor agonist or antagonist. The results showed that RVM contributes to restoration of cerebral perfusion following an SAH. Failure in RVM could lead to the acute perfusion deficits seen in patients following SAH.

Blood clots within the subarachnoid space impair cerebrospinal fluid (CSF) flow, which contributes to the increased ICP and impaired cortical perfusion during the early phase $(24 \mathrm{~h})$ after SAH. Siler et al. ${ }^{78}$ showed that intracisternal administration of tissue plasminogen activator (tPA) immediately after SAH lowered ICP, increased CBV, and partially restored CSF flow $24 \mathrm{~h}$ after SAH (Fig. 9). Lowering ICP without tPA, by draining CSF, improved CBV at $1 \mathrm{~h}$, but not $24 \mathrm{~h}$, after SAH. These findings suggest that blockade of CSF flow by microthrombi contributes to the early decline in cortical perfusion in an ICP-dependent and ICP-independent manner. Intracisternal tPA administration immediately after SAH partially restores CSF flow, reduces ICP, and improves cortical perfusion.

\section{Discussion and Outlook}

Accurate visualization and quantification of the microvascular networks in the cerebral cortex are critical for evaluating therapies that are aimed to treat neurovascular diseases, such as stroke, TBI, vascular dementia, inflammation, and cancer. The neuron firing rate changes within a wide range from $\sim 1$ to $200 \mathrm{~Hz}$, depending on the activity and which part of the brain is involved. ${ }^{79}$ This causes capillaries to exhibit heterogeneous and fluctuating RBC dynamics.

Intensity- or complex signal-based methods are superior to Doppler methods for in vivo visualization of capillaries in the cortex. However, RBC flux measurement in capillaries is still a challenging task using currently available methods. New methods applicable to OCT systems with improved development of both hardware and algorithms are needed. Combined OCT flux maps with OCT angiography images, including velocity measurement using the Doppler approach, would provide critical information for a comprehensive understanding of microvascular dynamics in the brain.

Although OCT technology has made great progress over the last decade, there are still some caveats. First, the lateral resolution of typical systems is $\sim 10 \mu \mathrm{m}$ with a depth of focus of $\sim 0.15 \mathrm{~mm}$. This makes diameter measurements inaccurate for those vessels smaller than $20 \mu \mathrm{m}$. This problem may be mitigated by a cost-effective higher-resolution system with sufficient depth of focus to cover the entire cortical layer. In addition, cranial window techniques can be implemented to increase the penetration depth and the quality of the OCT
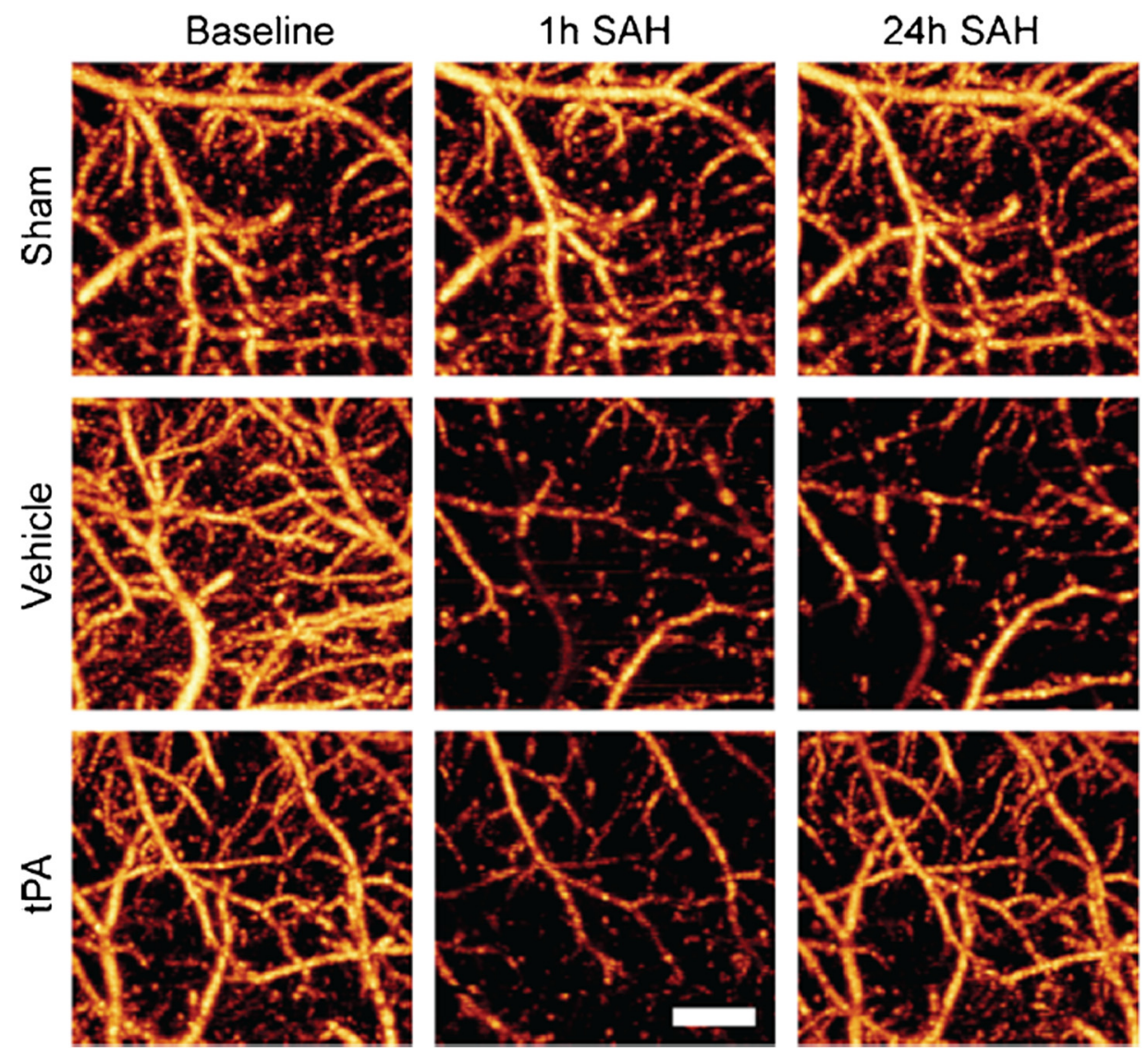

Fig. 9 Representative OCT angiography images of the cortex within the MCA territory at baseline, $1 \mathrm{~h}$ post-SAH/sham (pretreatment) and $24 \mathrm{~h}$ post-SAH/sham (posttreatment). ${ }^{78}$ tPA reduces ICP and improves cortical perfusion $24 \mathrm{~h}$ after $\mathrm{SAH}$. Scale bar $=500 \mu \mathrm{m}$. 
angiograms. ${ }^{80}$ An alternative solution would be to combine OCT with other imaging tools that can provide higher resolution, such as confocal microscopy and TPM. ${ }^{62}$ The combination of OCT with these techniques may provide additional information that is useful to investigate the vascular involvement in neurological diseases. One limitation of using other techniques would be the administration of exogenous contrast agents, which requires additional safety screening and can produce cytotoxic by-products.

Second, Doppler methods can measure only axial velocity and typically fail to detect the RBC velocities and the flow in the pial vessels perpendicular to the optical axis, due to their very small axial velocities. The absolute flow information is difficult to derive accurately, due to the resolution and the light intensity deterioration along the depth. Hence, current OCT angiography is best suited for comparative studies where errors in the measurements do not affect the differential conclusions.

Furthermore, the motion-induced noise can cause the quality of OCT angiograms to deteriorate significantly. Motion tracking systems should be employed for awake animal experiments. In addition, the $\sim 1.5$-mm penetration depth of OCT limits its applications to the cortex, typically with a cranial window. Luckily, the development of catheter probes using MEMS scanners ${ }^{81}$ is surging and it can extend the use of OCT in deeper layers of the brain.

SS-OCT systems eliminate the requirement of line-scan cameras that are needed in SD-OCT systems, and they can be implemented in the 1300-nm wavelength range where silicon-based detectors lack sensitivity. Overall, SS-OCT offers several advantages over SD-OCT, such as increased imaging range, decreased depth-dependent signal roll-off, and less motion-induced signal loss due to fringe washout. ${ }^{82}$ However, SS-OCT typically lacks phase stability and has a shorter life span. Recent advancements in SS lasers pushed the OCT speed into approximately the megahertz line rate era, ${ }^{83}$ and volumetric OCT microstructure and microvascular imaging at video rates has been demonstrated. ${ }^{12}$ At these high speeds, difficult-to-control resonant scanners have to be utilized instead of nonresonant galvo scanners to scan the probe beam. Moreover, the sensitivity of the system drops with the increase of system speed since the number of photons available for each A-line decreases with the increase of imaging speed. These lead to new challenges in achieving high-quality four-dimensional (4-D) OCT angiograms. However, 4-D OCT angiograms with high resolution capabilities would be a great help for understanding the ecosystem of a rapidly changing brain.

OCT angiography is a surging imaging modality that has applications for several clinical and basic research investigations. Although it has been witnessed as a revolutionary tool in clinical ophthalmology, OCT has not been widely adopted in neuroscience research yet. Given its high spatial resolution and velocity sensitivity, OCT angiography can provide useful information regarding highly complicated microcirculation dynamics in brain. We foresee that OCT angiography will become an indispensable, yet cost-effective, tool in the monitoring of changes in tissue morphology and hemodynamics following pharmacological interventions in small animal models.

\section{Acknowledgments}

This work was supported in part by National Institutes of Health grants (R01HL093140 and R01EB009682). The content is solely the responsibility of the authors and does not necessarily represent the official views of the grant-giving bodies. The effort in completing this review paper does not involve the use of animal and/or human subjects in the study. The results presented are cited from the published literature.

\section{References}

1. T. J. Vincent et al., "Longitudinal brain size measurements in APP/PS1 transgenic mice," Magn. Reson. Insights 4, 19-26 (2010).

2. D. P. Cardenas et al., "Functional MRI during hyperbaric oxygen: effects of oxygen on neurovascular coupling and BOLD fMRI signals," NeuroImage 119, 382-389 (2015).

3. C. Schaffer et al., "Two-photon imaging of cortical surface microvessels reveals a robust redistribution in blood flow after vascular occlusion," PLoS Biol. 4(2), e22 (2006).

4. C. A. Molina and J. L. Saver, "Extending reperfusion therapy for acute ischemic stroke: emerging pharmacological, mechanical, and imaging strategies," Stroke 36(10), 2311-2320 (2005).

5. D. Malonek, "Vascular imprints of neuronal activity: relationships between the dynamics of cortical blood flow, oxygenation, and volume changes following sensory stimulation," Proc. Natl. Acad. Sci. 94(26), 14826-14831 (1997).

6. D. A. Boas and A. K. Dunn, "Laser speckle contrast imaging in biomedical optics," J. Biomed. Opt. 15(1), 011109 (2010).

7. C. K. Willie et al., "Utility of transcranial Doppler ultrasound for the integrative assessment of cerebrovascular function," J. Neurosci. Methods 196(2), 221-237 (2011).

8. D. L. Johnson et al., "Cerebral blood flow and personality: a positron emission tomography study," Am. J. Psychiatry 156(2), 252-257 (1999).

9. B. A. Sutherland, T. Rabie, and A. M. Buchan, "Laser Doppler flowmetry to measure changes in cerebral blood flow," Cereb. Angiogenesis 1135, 237-248 (2014).

10. B. Ning et al., "Simultaneous photoacoustic microscopy of microvascular anatomy, oxygen saturation, and blood flow," Opt. Lett. 40(6), 910-913 (2015).

11. P. H. Tomlins and R. K. Wang, "Theory, developments and applications of optical coherence tomography," J. Phys. D 38(15), 2519-2535 (2005).

12. Z. Zhi et al., "4D optical coherence tomography-based micro-angiography achieved by 1.6-MHz FDML swept source," Opt. Lett. 40(8), 1779-1782 (2015).

13. W. Drexler et al., "In vivo ultrahigh-resolution optical coherence tomography," Opt. Lett. 24(17), 1221-1223 (1999).

14. M. Wojtkowski et al., "Ultrahigh-resolution, high-speed, Fourier domain optical coherence tomography and methods for dispersion compensation," Opt. Express 12(11), 2404-2422 (2004).

15. D. Huang et al., "Optical coherence tomography," Science 254(5035), 1178-1181 (1991).

16. R. Leitgeb, C. Hitzenberger, and A. Fercher, "Performance of Fourier domain vs. time domain optical coherence tomography," Opt. Express 11(8), 889-894 (2003).

17. B. J. Vakoc et al., "Three-dimensional microscopy of the tumor microenvironment in vivo using optical frequency domain imaging," Nat. Med. 15(10), 1219-1223 (2009).

18. S. A. Boppart, "Optical coherence tomography: technology and applications for neuroimaging," Psychophysiology 40(4), 529-541 (2003).

19. M. Choma et al., "Sensitivity advantage of swept source and Fourier domain optical coherence tomography," Opt. Express 11(18), 21832189 (2003).

20. M-T. Tsai et al., "Microvascular imaging using swept-source optical coherence tomography with single-channel acquisition," Appl. Phys. Express 4(9), 097001 (2011).

21. A. Zhang et al., "Methods and algorithms for optical coherence tomography-based angiography: a review and comparison," J. Biomed. Opt. 20(10), 100901 (2015).

22. R. K. Wang et al., "Three dimensional optical angiography," Opt. Express 15(7), 4083-4097 (2007).

23. A. S. Nam, I. Chico-Calero, and B. J. Vakoc, "Complex differential variance algorithm for optical coherence tomography angiography," Biomed. Opt. Express 5(11), 3822-3832 (2014). 
24. L. An, J. Qin, and R. K. Wang, "Ultrahigh sensitive optical microangiography for in vivo imaging of microcirculations within human skin tissue beds," Opt. Express 18(8), 8220-8228 (2010).

25. Y. Wang and R. Wang, "Autocorrelation optical coherence tomography for mapping transverse particle-flow velocity," Opt. Lett. 35(21), 35383540 (2010).

26. S. Yousefi and R. K. Wang, "Simultaneous estimation of bidirectional particle flow and relative flux using MUSIC-OCT: phantom studies," Phys. Med. Biol. 59(22), 6693-6708 (2014).

27. R. K. Wang and L. An, "Multifunctional imaging of human retina and choroid with 1050-nm spectral domain optical coherence tomography at 92-kHz line scan rate," J. Biomed. Opt. 16(5), 050503 (2011).

28. V. J. Srinivasan et al., "Rapid volumetric angiography of cortical microvasculature with optical coherence tomography," Opt. Lett. 35(1), 43-45 (2010).

29. Z. Chen et al., "Noninvasive imaging of in vivo blood flow velocity using optical Doppler tomography," Opt. Lett. 22(14), 1119-1121 (1997).

30. S. Makita et al., "Optical coherence angiography," Opt. Express 14(17), 7821-7840 (2006).

31. K. Kurokawa et al., "Three-dimensional retinal and choroidal capillary imaging by power Doppler optical coherence angiography with adaptive optics," Opt. Express 20(20), 22796-22812 (2012).

32. D. Y. Kim et al., "In vivo volumetric imaging of human retinal circulation with phase-variance optical coherence tomography," Biomed. Opt. Express 2(6), 1504-1513 (2011).

33. G. Liu et al., "A comparison of Doppler optical coherence tomography methods," Biomed. Opt. Express 3(10), 2669-2680 (2012).

34. J. You et al., "Optical coherence Doppler tomography for quantitative cerebral blood flow imaging," Biomed. Opt. Express 5(9), 3217-3230 (2014).

35. R. K. Wang and L. An, "Doppler optical micro-angiography for volumetric imaging of vascular perfusion in vivo," Opt. Express 17(11), 8926-8940 (2009).

36. L. Shi et al., "Wide velocity range Doppler optical microangiography using optimized step-scanning protocol with phase variance mask," J. Biomed. Opt. 18(10), 106015 (2013).

37. R. K. Wang and Z. Ma, "Real-time flow imaging by removing texture pattern artifacts in spectral-domain optical Doppler tomography," Opt. Lett. 31(20), 3001-3003 (2006).

38. S. H. Yun et al., "Motion artifacts in optical coherence tomography with frequency-domain ranging," Opt. Express 12(13), 2977-2998 (2004).

39. E. Jonathan, J. Enfield, and M. J. Leahy, "Correlation mapping method for generating microcirculation morphology from optical coherence tomography (OCT) intensity images," J. Biophotonics. 4(9), 583-587 (2011).

40. Y. Jia et al., "Split-spectrum amplitude-decorrelation angiography with optical coherence tomography," Opt. Express 20(4), 4710-4725 (2012).

41. A. Mariampillai et al., "Optimized speckle variance OCT imaging of microvasculature," Opt. Lett. 35(8), 1257-1259 (2010).

42. W. J. Choi et al., "Improved microcirculation imaging of human skin in vivo using optical microangiography with a correlation mapping mask," J. Biomed. Opt. 19(3), 036010 (2014).

43. J. Barton and S. Stromski, "Flow measurement without phase information in optical coherence tomography images," Opt. Express 13(14), 5234-5239 (2005).

44. H. Ren et al., "Quantitative imaging of red blood cell velocity invivo using optical coherence Doppler tomography," Appl. Phys. Lett. 100(23), 233702 (2012).

45. J. Lee et al., "Multiple-capillary measurement of RBC speed, flux, and density with optical coherence tomography," J. Cereb. Blood Flow Metab. 33(11), 1707-1710 (2013).

46. J. Lee et al., "Statistical intensity variation analysis for rapid volumetric imaging of capillary network flux," Biomed. Opt. Express 5(4), 11601172 (2014).

47. O. Krichevsky and G. Bonnet, "Fluorescence correlation spectroscopy: the technique and its applications," Rep. Prog. Phys. 65(2), 251 (2002).

48. Y. Wang and R. K. Wang, "Measurement of particle concentration in flow by statistical analyses of optical coherence tomography signals," Opt. Lett. 36(11), 2143-2145 (2011).

49. V. J. Srinivasan et al., "OCT methods for capillary velocimetry," Biomed. Opt. Express 3(3), 612-629 (2012).
50. R. V. Harrison, "Blood capillary distribution correlates with hemodynamic-based functional imaging in cerebral cortex," Cereb. Cortex. 12(3), 225-233 (2002).

51. R. K. Wang and S. Hurst, "Mapping of cerebro-vascular blood perfusion in mice with skin and skull intact by optical micro-angiography at $1.3 \mu \mathrm{m}$ wavelength," Opt. Express 15(18), 11402-11412 (2007).

52. Y. Jia, L. An, and R. K. Wang, "Label-free and highly sensitive optical imaging of detailed microcirculation within meninges and cortex in mice with the cranium left intact," J. Biomed. Opt. 15(3), 030510 (2010).

53. Y. Jia and R. K. Wang, "Label-free in vivo optical imaging of functional microcirculations within meninges and cortex in mice," J. Neurosci. Methods 194(1), 108-115 (2010).

54. Y. Jia, P. Li, and R. K. Wang, "Optical microangiography provides an ability to monitor responses of cerebral microcirculation to hypoxia and hyperoxia in mice," J. Biomed. Opt. 16(9), 096019 (2011).

55. V. J. Srinivasan et al., "Quantitative cerebral blood flow with optical coherence tomography," Opt. Express 18(3), 2477-2494 (2010).

56. R. W. Gill, "Measurement of blood flow by ultrasound: accuracy and sources of error," Ultrasound Med. Biol. 11(4), 625-641 (1985).

57. Y. Nakao et al., "Effects of anesthesia on functional activation of cerebral blood flow and metabolism," Proc. Natl. Acad. Sci. U.S.A. 98(13), 7593-7598 (2001).

58. V. J. Srinivasan et al., "Optical coherence tomography for the quantitative study of cerebrovascular physiology," J. Cereb. Blood Flow Metab. 31(6), 1339-1345 (2011).

59. D. Kleinfeld et al., "Fluctuations and stimulus-induced changes in blood flow observed in individual capillaries in layers 2 through 4 of rat neocortex," Proc. Natl. Acad. Sci. U.S.A. 95(26), 15741-15746 (1998).

60. B. Stefanovic et al., "Functional reactivity of cerebral capillaries," J. Cereb. Blood Flow Metab. 28(5), 961-972 (2008).

61. S. Dziennis et al., "Macro-to-micro cortical vascular imaging underlies regional differences in ischemic brain," Sci. Rep. 5, 10051 (2015).

62. L. Gagnon et al., "Multimodal reconstruction of microvascular-flow distributions using combined two-photon microscopy and Doppler optical coherence tomography," Neurophotonics 2(1), 015008 (2015).

63. H. Wang et al., "Does optical microangiography provide accurate imaging of capillary vessels? Validation using multiphoton microscopy," J. Biomed. Opt. 19(10), 106011 (2014).

64. K. Sampei et al., "Stroke in estrogen receptor- $\alpha$-deficient mice," Stroke 31(3), 738-744 (2000).

65. Y. Jia and R. K. Wang, "Optical micro-angiography images structural and functional cerebral blood perfusion in mice with cranium left intact," J. Biophotonics 4(1-2), 57-63 (2011).

66. V. J. Srinivasan et al., "Multiparametric, longitudinal optical coherence tomography imaging reveals acute injury and chronic recovery in experimental ischemic stroke," PLoS One 8(8), e71478 (2013).

67. L. Yu et al., "Spectral Doppler optical coherence tomography imaging of localized ischemic stroke in a mouse model," J. Biomed. Opt. 15(6), 066006 (2010).

68. M. A. Berny-Lang et al., "Thrombin mutant W215A/E217A treatment improves neurological outcome and reduces cerebral infarct size in a mouse model of ischemic stroke," Stroke 42(6), 1736-1741 (2011).

69. C. Sun et al., "Conditional ablation of neuroprogenitor cells in adult mice impedes recovery of poststroke cognitive function and reduces synaptic connectivity in the perforant pathway," J. Neurosci. 33(44), 17314-17325 (2013).

70. Y. Akamatsu et al., "Impaired leptomeningeal collateral flow contributes to the poor outcome following experimental stroke in the type 2 diabetic mice," J. Neurosci. 35(9), 3851-3864 (2015).

71. U. Baran, Y. Li, and R. K. Wang, "Vasodynamics of pial and penetrating arterioles in relation to arteriolo-arteriolar anastomosis after focal stroke," Neurophotonics 2(2), 025006 (2015).

72. C. Werner and K. Engelhard, "Pathophysiology of traumatic brain injury," Br. J. Anaesthesia 99(1), 4-9 (2007).

73. Y. Jia, N. Alkayed, and R. K. Wang, "Potential of optical microangiography to monitor cerebral blood perfusion and vascular plasticity following traumatic brain injury in mice in vivo," J. Biomed. Opt. 14(4), 040505 (2009). 
74. J. S. Cetas et al., "Brainstem control of cerebral blood flow and application to acute vasospasm following experimental subarachnoid hemorrhage," Neuroscience 163(2), 719-729 (2009).

75. Y. Jia et al., "In vivo optical imaging of revascularization after brain trauma in mice," Microvasc. Res. 81(1), 73-80 (2011).

76. C. E. Johanson et al., "Multiplicity of cerebrospinal fluid functions: new challenges in health and disease," Cerebrospinal Fluid Res. 5(10), 441-450 (2008).

77. J. van Gijn, R. S. Kerr, and G. J. E. Rinkel, "Subarachnoid haemorrhage," Lancet 369(9558), 306-318 (2007).

78. D. A. Siler et al., "Intracisternal administration of tissue plasminogen activator improves cerebrospinal fluid flow and cortical perfusion after subarachnoid hemorrhage in mice," Transl. Stroke Res. 5(2), 227-237 (2014).

79. J. J. Harris, R. Jolivet, and D. Attwell, "Synaptic energy use and supply," Neuron 75(5), 762-777 (2012).

80. Y. Li, U. Baran, and R. K. Wang, "Application of thinned-skull cranial window to mouse cerebral blood flow imaging using optical microangiography," PLoS One 9(11), e113658 (2014).

81. S. T. S. Holmstrom, U. Baran, and H. Urey, "MEMS laser scanners: a review," J. Microelectromech. Syst. 23(2), 259-275 (2014).

82. B. Potsaid et al., "Ultrahigh speed $1050 \mathrm{~nm}$ swept source/Fourier domain OCT retinal and anterior segment imaging at 100,000 to 400,000 axial scans per second," Opt. Express 18(19), 20029-20048 (2010).
83. W. Wieser et al., "Multi-megahertz OCT: high quality 3D imaging at 20 million A-scans and 4.5 GVoxels per second," Opt. Express. 18(14), 14685-14704 (2010).

Utku Baran received his BS and MS degrees in electrical engineering from Koc University, Istanbul, Turkey, in 2010 and 2012, respectively. $\mathrm{He}$ is currently a PhD candidate in the Department of Electrical Engineering at the University of Washington, Seattle. He is the author/coauthor of more than 16 journal articles, and the coinventor of three pending U.S. patents. His interests include MEMS, displays, biomedical optics, and neuroscience. He is a recipient of SPIE Optics and Photonics Education Scholarship and Newport Research Excellence Travel Award.

Ruikang Wang is currently a professor of bioengineering and ophthalmology at the University of Washington and directs the Biophotonics and Imaging Laboratory. He is a fellow of Optical Society of America (OSA), SPIE and American Institute for Medical and Biological Engineering (AIMBE). His current research interests include biophotonics and imaging, optical coherence tomography, optical microangiography, photoacoustic imaging and their applications in neurology, ophthalmology, dermatology, and cancer. 